\title{
DNA-tethered Polymersome Clusters as Nanotheranostic Platform
}

\author{
Claire E. Meyer ${ }^{\S}$, Cora-Ann Schoenenberger, Juan Liu, Ioana Craciun, and Cornelia G. Palivan*
}

§SCS-Metrohm award for best oral presentation in Polymers, Colloids \& Interfaces

\begin{abstract}
Nanotheranostics combine the use of nanomaterials and biologically active compounds to achieve diagnosis and treatment at the same time. To date, severe limitations compromise the use of nanotheranostic systems as potent nanomaterials are often incompatible with potent biomolecules. Herein we emphasize how a novel type of polymersome clusters loaded with active molecules can be optimized to obtain an efficient nanotheranostic platform. Polymersomes loaded with enzymes and specific dyes, respectively and exposing complementary DNA strands at their external surface formed clusters by means of DNA hybridization. We describe factors at the molecular level and other conditions that need to be optimized at each step of the cluster formation to favor theranostic efficiency.
\end{abstract}

Keywords: Cluster · DNA tethering · Enzymes · Nanotheranostics · Polymersome

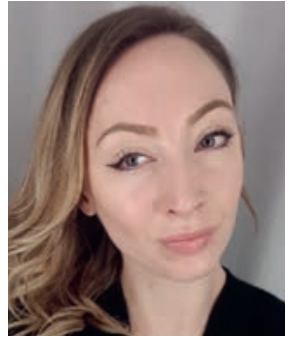

Claire Elsa Meyer completed a 2-year technology training in Materials Science and Engineering before receiving her Master's degree in Chemistry and Biology from the University of Strasbourg, France. She did two research internships in Strasbourg, at the Institut National de la Santé et de la Recherche en Médecine (INSERM) and at the Institut de Science et d'Ingénierie Supramoléculaire, where she studied and designed biological macromolecules for biomedical applications. She continued to focus on the development of complex biohybrid constructs when she joined the group of Prof. Cornelia Palivan at the Department of Chemistry of the University of Basel, Switzerland, where she is working on injectable polymersomebased nanodevices for therapeutic, imaging and theranostic applications. Her work was recently recognized by one of the 2020 Swiss Nanotechnology PhD Awards and she was granted a Swiss National Science Foundation (SNSF) Early Postdoc Mobility Fellowship to pursue her research at the University of California, Los Angeles (UCLA), USA.

\section{Introduction}

Theranostics is a field of medicine that combines both therapy and diagnosis to reduce multi-step procedures thereby improving patient care and enhancing chances of cure. The therapeutic part of theranostic systems is usually achieved via release of bioactive compounds, hyperthermia or reactive organic species (ROS) production, while the diagnosis part mostly involves imaging methods like MRI, fluorescence or ultrasound.[1] Theranostic systems are often based on nanomaterials whose small size and interesting intrinsic properties make them particularly suited for such dual applications. For example, inorganic nanoparticles like SPION or quantum dots possess the unique advantage of being intrinsic imaging agents but their potential toxicity limits application. ${ }^{[1,2]}$ On the other hand, lipid- or polymer-based micelles are potent support materials as they are non-toxic and can integrate hydrophobic therapeutic/imaging agents within their hydrophobic core. ${ }^{[1,3]}$ However, increasing the range of applications crucially depends on improving the loading capacity for hydrophilic active compounds which cannot be achieved by using micelles. Of particular interest are soft nanocompartments, i.e. liposomes and polymersomes as they consist of an amphiphilic membrane enclosing an aqueous cavity and therefore allow for integrating hydrophobic compounds within their membrane and loading their cavity with hydrophilic compounds. ${ }^{[4-6]}$ In addition, the external surface of nanocompartments can be functionalized to gain targeting properties, especially in the case of polymersomes as they benefit from the sheer endless chemical versatility of polymers. ${ }^{[4]}$ As polymersomes have an increased mechanical stability compared to liposomes, they appear to be ideal materials for building a non-toxic nanotheranostic platform, adaptable to different applications..$^{[4,5]}$ The architecture of nanothernostic systems usually comprises assemblies simultaneously containing therapeutic and imaging compounds, for example via co-encapsulation of both agents inside soft-nanocompartments. ${ }^{[7]}$ However, co-encapsulation can be detrimental for sensitive compounds or negatively affect the encapsulation efficiency of active agents. These serious drawbacks can be overcome by another type of nanotheranostic architecture based on super-assemblies: the combination of distinct nanomaterials that each are equipped with either the therapeutic or the imaging function. However, most super-assemblies are composed of inorganic nanoparticles and/or held together by weak, non-covalent interactions that compromise their efficacy, especially under physiological conditions where dissociation of the assembly is likely to occur. ${ }^{[8-10]}$ To overcome such constraints, we developed a super-assembly composed of two different types of polymersomes, one bearing the therapeutic function while the other one enables imaging, tethered together by hybridization of complementary DNA strands exposed on the respective surfaces (Fig. 1a). ${ }^{[11,12]}$ In addition, the ssDNA not involved in tethering served to promote attachment to cells expressing scavenger 
receptors, e.g. epithelial cells. ${ }^{[13]}$ In view of the enzyme (Dopa Decarboxylase, DCC) encapsulated into the therapeutic polymersomes, our system is geared towards applications in the treatment of atherosclerosis in that polymersome clusters could attach to atherosclerotic lesions, produce and deliver on-site a therapeutic compound (dopamine), while at the same time, the imaging compartment serves to follow the lesions' dynamics by the imaging modality. ${ }^{[11]}$ In general, the imaging property of theranostic systems is achieved via encapsulation of fluorescent dyes or MRI agents within the 'imaging' polymersome. ${ }^{[1,4,5,11]}$ The communication between the external environment and the polymersome cavity where enzymes were located, was accomplished by permeabilization of the polymer membrane via incorporation of pore forming proteins (Outer membrane protein F, OmpF). ${ }^{[14,15]}$ This way, substrates reached the encapsulated enzymes and the resulting therapeutic product diffused out of the polymersomes to act on nearby cells. ${ }^{[11]}$ These polymersome clusters offer great advantages such as robustness, stability in physiological environments, and in vivo, non-toxicity, efficient dual-functionality (due to the segregation of the therapeutic and imaging compounds), and can be easily modified to achieve desired applications. ${ }^{[4,11,13]}$ To accommodate the therapeutic enzymes or imaging agents chosen in view of a specific application, several steps of the polymersome cluster formation need to be optimized. Here, we explain how the optimization steps were achieved for polymersome clusters at a molecular level (Fig. 1b), in respect to the conditions required to preserve the nanocompartments' architecture, to support their clustering and ultimately obtain the dual-functionality required for theranostic applications.

\section{Optimization of Polymersome Clusters forTheranostic Applications}

\subsection{Nanotheranostic Polymersome Cluster Formation}

Nanotheranostic polymersome clusters are formed during a four-step procedure: 1) thin polymer film formation, 2) polymersome formation by self-assembly, 3) ssDNA coupling to the external surface of polymersomes and 4) clustering of loaded polymersomes into super-assemblies (Fig. 1b). Each of these steps has to be optimized in different ways for a given application. To prepare polymersomes by the film-rehydration method, a solution of amphiphilic copolymers is dried in a round-bottom flask to form a thin film of polymer which is then rehydrated with an aqueous solution containing active compounds (enzymes or imaging agents) that get encapsulated while the polymer film detaches from the flask and self-assembles into nano-sized assemblies. ${ }^{[1]}$ The therapeutic and imaging polymersomes were prepared in parallel, each under specific conditions, before being surface-functionalized with complementary ssDNA. After removal of unbound ssDNA and non-encapsulated active compounds, both kinds of polymersome were mixed together to induce DNA hybridization for cluster formation. ${ }^{[11-13]}$

\subsection{Optimization of Polymersome Formation}

When selecting a copolymer, requirements in terms of the self-assembly process that should yield polymersomes and provide an enhanced functionalization potential have to be taken into account. In this respect, we used a diblock copolymer poly (dimethylsiloxane)-block-poly(2-methyloxazoline) (PMOXA- $b$ - a)

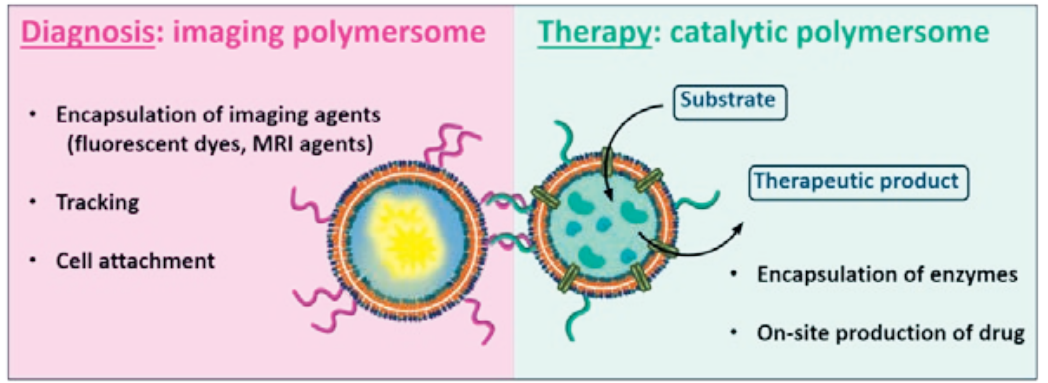

b)

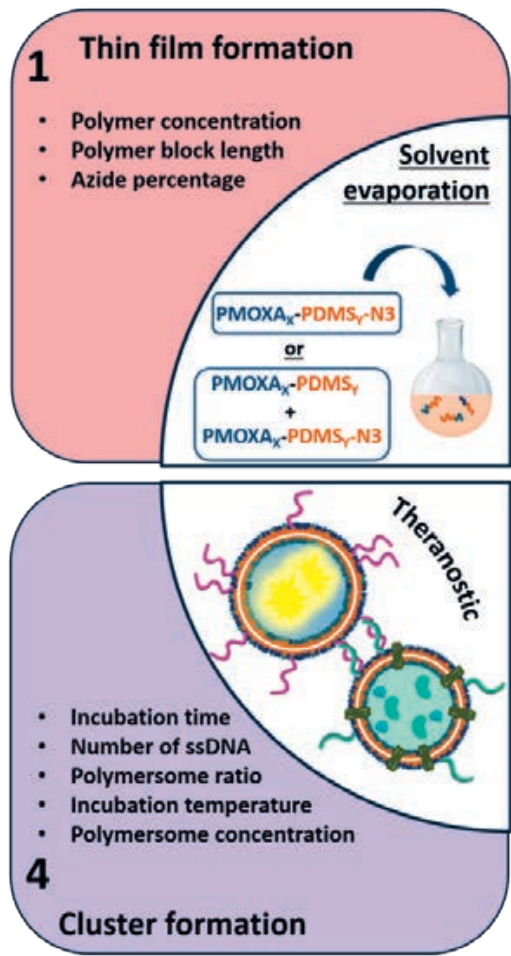

Polymersome formation
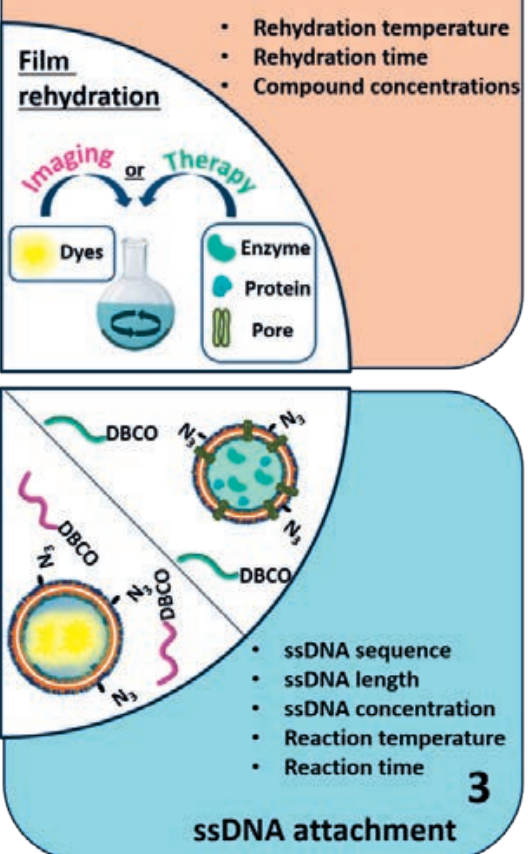

Fig. 1. Nanotheranostic polymersome clusters: (a) architecture providing therapeutic and diagnostic features (b) four steps involved in nanocluster formation with corresponding optimization possibilities. 
PDMS) known to generate polymersomes by self-assembly in dilute aqueous solutions. ${ }^{[11,16]}$ The ability of the copolymer to readily self-assemble into polymersomes is determined by the hydrophilic-to-hydrophobic ratio of the respective blocks. We selected rather short copolymer block lengths $\left(\mathrm{PDMS}_{22}-b\right.$-PMOXA $)$ to favor polymersome formation. ${ }^{[11]}$ The non-functionalized $\mathrm{PDMS}_{22}{ }^{-}$ $b$-PMOXA ${ }_{8}$ copolymers were mixed with reactive azide-functionalized copolymers $\left(\mathrm{PDMS}_{22}-b-\mathrm{PMOXA}_{8}-\mathrm{OEG}_{3}-\mathrm{N}_{3}\right.$ ) prior to the thin film formation. To facilitate surface functionalization of formed polymersomes while preserving the membrane integrity, the fraction of azide-copolymer in the polymer mixture was varied from 1 to $100 \% .^{[11-13]}$ In addition, the total concentration of polymer used for film formation was varied to obtain concentrated polymersome solutions but avoid aggregation. ${ }^{[11]}$ Here, we emphasize that almost five-times more polymersomes were formed at a polymer concentration of $10 \mathrm{mg} / \mathrm{mL}$ compared to $2 \mathrm{mg} / \mathrm{mL}$ (Table 1). It is essential to achieve high polymersome concentrations for an improved therapeutic and imaging efficiency, especially since after both kinds of polymersome are prepared in parallel, they are mixed together (in 1:1 ratio) to form the clusters, resulting in halved respective concentrations. ${ }^{[11]}$ Taken together, the choice of the copolymer and the accessibility of azide groups are crucial and have considerable effects on the rest of the cluster formation procedure.

In the second step -the polymersome formation via the film rehydration method, it was critical to use a short copolymer not only for self-assembly to occur very fast (less than one minute at room temperature) and at a broad range of temperatures $\left(4^{\circ} \mathrm{C}-\right.$ $>25^{\circ} \mathrm{C}$ ) but also to achieve protein insertion into the membrane. [11] Depending on the specificity of the enzymes and dyes to be encapsulated (solubility, stability), the conditions of the self-assembly process have to be adapted to preserve the functionality of the active compounds, which is critical to achieve efficient theranostic response. Therefore, thanks to the short length of our polymer, the conditions of vesicle formation $\left(3\right.$ hours at $4{ }^{\circ} \mathrm{C}$ ) had been tuned to support the encapsulation of compounds (enzymes, proteins, fluorescent dyes) without affecting their properties. The encapsulation via film rehydration is a statistical process which implies that the amount of encapsulated compound is not linearly correlated with the compound concentration of the rehydration solution and therefore, the encapsulation efficiency often remains relatively low. ${ }^{[17]}$ However, the limited compound encapsulation (enzymes, imaging agents) was not an issue as we selected compounds with efficient therapeutic/imaging properties and performed their encapsulation under adapted conditions. ${ }^{[11,16,18]}$

\subsection{Optimization of Cluster Formation}

The third step of polymersome cluster formation consists of grafting complementary ssDNA onto the surface of the different polymersomes. We used azide-alkyne cycloaddition (SPAAC)
Table 1. Number of polymersomes per $\mathrm{mL}$ obtained from the rehydration of $600 \mu \mathrm{L}$ of PDMS22- $b$-PMOXA8 with PBS for $12 \mathrm{~h}$ at room temperature, at different concentrations.

\begin{tabular}{|l|l|l|}
\hline $\begin{array}{l}\text { Concentration } \\
\text { of polymer }\end{array}$ & $2 \mathrm{mg} / \mathrm{mL}$ & $10 \mathrm{mg} / \mathrm{mL}$ \\
\hline $\begin{array}{l}\text { Number of } \\
\text { polymersomes }\end{array}$ & $4.8 \times 10^{11} / \mathrm{mL}$ & $2.1 \times 10^{12} / \mathrm{mL}$ \\
\hline
\end{tabular}

with dibenzocyclooctyne DBCO-functionalized ssDNA that reacts with azide groups exposed by the polymersome surface. ${ }^{[12]}$ To preserve the functionality of encapsulated active compounds, the surface functionalization of polymersomes with ssDNA was performed at low temperature $\left(4^{\circ} \mathrm{C}\right) \cdot{ }^{[11]}$ To facilitate the SPAAC reaction, ssDNA sequences should be chosen such as to avoid the formation of secondary structures, e.g. hairpins that might interfere with the availability of the DBCO group. Besides, the length and sequence of the ssDNA were tailored to promote cell attachment of clusters. ${ }^{[11,13]}$ After the hybridization of DNA between 'complementary' polymersomes, which induced cluster formation (see below), ${ }^{[12]}$ around $80 \%$ of the total number of ssDNA remained exposed at the surface of the clusters. These residual single-stranded DNAs served for cell attachment as their negative charge exhibited a strong affinity for scavenger receptors that are highly expressed on distinct cell types. ${ }^{[19,20]}$

For the last step of cluster formation, polymersomes loaded with enzyme and dye, respectively, were mixed together and the hybridization of the complementary ssDNA resulted in their tethering into super-assemblies. In order to avoid aggregation and control the size of polymersome clusters, diverse factors have been varied: the ssDNA hybridization conditions (time and temperature), the concentration and the ratio of polymersomes with regard to the number of ssDNA present on each type of polymersome. ${ }^{[11-13]}$ For example, when one type of polymersome exposed significantly higher numbers of ssDNA on its surface (around 100-200 ssDNA/polymersome) compared to the other type of polymersome (20-30 ssDNA/polymersome), the formation of clusters at $20{ }^{\circ} \mathrm{C}$ was still possible because the probability of annealing was still high enough due to high surface density of at least one kind of ssDNA strand. ${ }^{[11]}$ In contrast, when both polymersomes have a low surface density of ssDNA, no clusters were formed. On the other hand, if both types of polymersomes expose high densities of ssDNA they tend to aggregate, which was avoided by diluting the polymersome solutions and/or decreasing the temperature (no cluster formation was observed at $4{ }^{\circ} \mathrm{C}$ ). ${ }^{[11]}$ Taken together, the optimization of the surface density of ssDNA/polymersome and the conditions for promoting hybridization have to be specifically
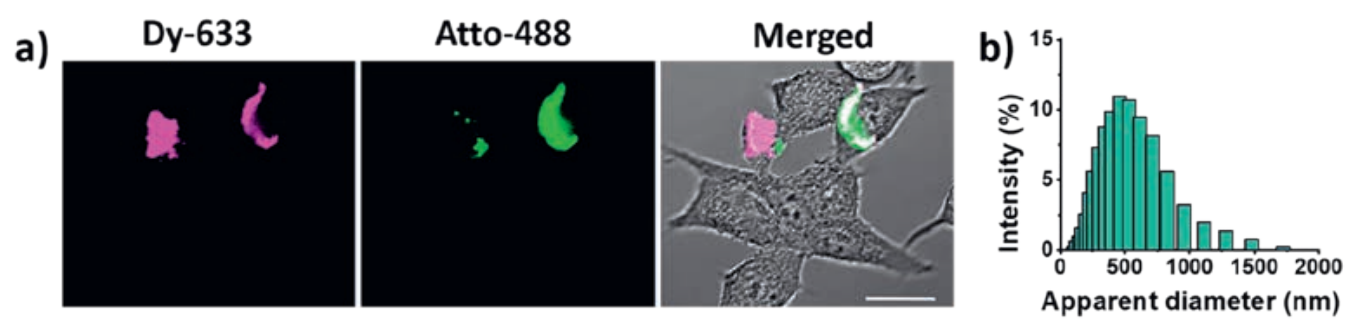

Fig. 2. a) Confocal laser scanning microscopy images of model clusters composed of two kinds of polymersomes encapsulating fluorescent dyes (Dy-633 or Atto488) reveal the co-localization of both types of fluorescent polymersomes within clusters and their attachment to the surface of scavenger receptor expressing HEK293T cells (scale bar $=20$ $\mu \mathrm{m})$. b) Dynamic light scattering (DLS) showing the size of nanotheranostic polymersome clusters after one year of storage in PBS at $4{ }^{\circ} \mathrm{C}$. 
adjusted to avoid aggregation and obtain clusters with a desired size. ${ }^{[11-13]}$

Once the colloidal stability and controlled architecture of the clusters had been achieved, their dual-functionality was evaluated: by encapsulation of Dopa Decarboxylase (DDC) in the therapeutic polymersomes and Atto- 488 dye in the imaging ones, the clusters successfully produced dopamine in sufficient amounts to induce a cell response while generating a fluorescent signal for cell imaging. ${ }^{[11]}$ In addition, as illustrated in Fig. 2, nanotheranostic clusters dock onto cell membranes via interactions of ssDNA with scavenger receptors, further emphasizing the applicability for cell-specific interactions (Fig. 2a) and are stable upon longterm storage as clusters maintained their architecture and size $(495 \pm 212 \mathrm{~nm})$ for more than a year after formation (Fig. 2b), which is another essential feature for translational applications.

\section{Conclusions and Outlook}

Based on the many possibilities of adaptation, the superassembly of DNA-linked polymersomes loaded with different active compounds (enzymes for therapeutic purposes and dyes for imaging) represents a nanotheranostic platform that can be tailored to specific applications. Being constructed in a modular manner, these clusters offer extensive flexibility by an association of different polymersomes that can be tuned by a straightforward change of the enzymes or the contrast agents to a large variety of pathological conditions. The stable architecture makes these nanosystems particularly suited for implementing this technology with additional modalities including targeting, responsiveness to pathological stimuli and synergistic therapies.

\section{Acknowledgements}

The authors acknowledge the Swiss National Science Foundation, NCCR-MSE and the University of Basel for financial support. CEM thanks the Swiss Chemical Society and Metrohm for sponsoring the best oral presentation awards.

Received: January 29, 2021

[1] E.-K. Lim, T. Kim, S. Paik, S. Haam, Y.-M. Huh, K. Lee, Chem. Rev. 2015 115, 327, https://doi.org/10.1021/cr300213b

[2] J. Wang, X. Wang, Y. Song, J. Wang, C. Zhang, C. Chang, J. Yan, L. Qiu, M. Wu, Z. Guo, Chem. Sci. 2013, 4, 2605, https://doi.org/10.1039/C3SC50554E

[3] J. R. Upponi, K. Jerajani, D. K. Nagesha, P. Kulkarni, S. Sridhar, C. Ferris, V. P. Torchilin, Biomaterials 2018, 170, 26, https://doi.org/10.1016/j.biomaterials.2018.03.054
[4] C. E. Meyer, S.-L. Abram, I. Craciun, C. G. Palivan, Phys. Chem. Chem. Phys. 2020, 22, 11197, https://doi.org/10.1039/D0CP00693A

[5] L. Zartner, M. S. Muthwill, I. A. Dinu, C.-A. Schoenenberger, C. G. Palivan, J. Mater. Chem. B 2020, 8, 6252, https://doi.org/10.1039/D0TB00475H

[6] W. Lee, H.-J. Im, Nucl. Med. Mol. Imaging 2019, 53, 242, https://doi.org/10.1007/s13139-019-00603-z

[7] M. Mohammadi, M. Ramezani, K. Abnous, M. Alibolandi, Int. J. Pharm. 2017, 519, 287, https://doi.org/10.1016/j.ijpharm.2017.01.037

[8] N. Yang, T.-T. You, X. Liang, C.-M. Zhang, L. Jiang, P.-G. Yin, RSC Adv 2017, 7, 9321, https://doi.org/10.1039/C6RA27185E

[9] X. J. Loh, T.-C. Lee, Q. Dou, G. R. Deen, Biomater. Sci. 2016, 4, 70, https://doi.org/10.1039/C5BM00277J

[10] J.-B. Kim, K. Urban, E. Cochran, S. Lee, A. Ang, B. Rice, A. Bata, K. Campbell, R. Coffee, A. Gorodinsky, Z. Lu, H. Zhou, T. K. Kishimoto, P. Lassota, PLoS ONE 2010, 5, https://doi.org/10.1371/journal.pone.0009364

[11] C. E. Meyer, J. Liu, I. Craciun, D. Wu, H. Wang, M. Xie, M. Fussenegger, C. G. Palivan, Small 2020, 16, 1906492 https://doi.org/10.1002/smll.201906492

[12] J. Liu, V. Postupalenko, S. Lörcher, D. Wu, M. Chami, W. Meier, C. G. Palivan, Nano Lett. 2016, 16, 7128, https://doi.org/10.1021/acs.nanolett.6b03430

[13] J. Liu, I. Craciun, A. Belluati, D. Wu, S. Sieber, T. Einfalt, D. Witzigmann, M. Chami, J. Huwyler, C. G. Palivan, Nanoscale 2020, 12, 9786, https://doi.org/10.1039/D0NR00361A

[14] F. Itel, A. Najer, C. G. Palivan, W. Meier, Nano Lett. 2015, 15, 3871, https://doi.org/10.1021/acs.nanolett.5b00699

[15] A. Belluati, I. Craciun, C. E. Meyer, S. Rigo, C. G. Palivan, Curr. Opin. Biotechnol. 2019, 60, 53, https://doi.org/10.1016/j.copbio.2018.12.011

[16] C. E. Meyer, I. Craciun, C.-A. Schoenenberger, R. Wehr, C. G. Palivan, Nanoscale 2021, 13, 66, https://doi.org/10.1039/D0NR07178A

[17] Q. Chen, H. Schönherr, G. J. Vancso, Small 2009, 5, 1436, https://doi.org/10.1002/smll.200801455

[18] A. Belluati, I. Craciun, C. G. Palivan, ACS Nano 2020, 14, 12101 , https://doi.org/10.1021/acsnano.0c05574

[19] P. C. Patel, D. A. Giljohann, W. L. Daniel, D. Zheng, A. E. Prigodich, C. A. Mirkin, Bioconjug. Chem. 2010, 21, 2250, https://doi.org/10.1021/bc1002423

[20] N. Platt, S. Gordon, Chem. Biol. 1998, 5, R193, https://doi.org/10.1016/s1074-5521(98)90156-9

License and Terms

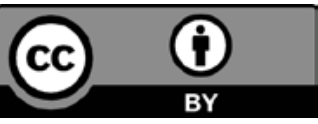

This is an Open Access article under the terms of the Creative Commons Attribution License CC BY 4.0. The material may not be used for commercial purposes.

The license is subject to the CHIMIA terms and conditions: (http:// chimia.ch/component/sppagebuilder/?view=page \&id=12).

The definitive version of this article is the electronic one that can be found at https://doi.org/10.2533/chimia.2021.296 